\title{
Relaxation mechanism in NiFe thin films driven by spin angular momentum absorption throughout the antiferromagnetic phase transition in native surface oxides
}

\author{
L. Frangou, ${ }^{1}$ G. Forestier, ${ }^{1}$ S. Auffret, ${ }^{1}$ S. Gambarelli, ${ }^{2}$ and V. Baltz ${ }^{1, *}$ \\ ${ }^{1}$ SPINTEC, Univ. Grenoble Alpes/CNRS/INAC-CEA, F-38000 Grenoble, France \\ ${ }^{2}$ SYMMES, Univ. Grenoble Alpes/INAC-CEA, F-38000 Grenoble, France \\ (Received 9 December 2016; revised manuscript received 26 January 2017; published 14 February 2017)
}

\begin{abstract}
We report an alternative mechanism for the physical origin of the temperature-dependent ferromagnetic relaxation observed in bare permalloy $(\mathrm{NiFe})$ thin films. Through spin-pumping experiments, we demonstrate that the peak in the temperature dependence of NiFe damping can be understood in terms of enhanced absorption of spin angular momentum at the magnetic phase transition in native antiferromagnetic surface-oxidized layers. These results suggest some avenues for the investigation of an incompletely understood phenomenon in physics.
\end{abstract}

DOI: 10.1103/PhysRevB.95.054416

In physical systems, damping characterizes the losses associated with out-of-equilibrium vibration dynamics [1,2]. In the field of spintronics, which relies on the spin-dependent transport properties of matter [3-5], magnetic damping is one of the key parameters as it regulates oscillations and switches in magnetization direction in any magnetic material $[6,7]$. Magnetic damping plays this role with all kinds of stimuli, whether the dynamics of magnetization is excited through an electromagnetic wave [8], an electrical current [9], or a spin current [10]. Damping in typical ferromagnetic materials has been thoroughly experimentally characterized through measurements of ferromagnetic resonance spectra and determination of their linewidths [11]. Several mechanisms have been proposed to explain these findings [11]. However, the basic mechanisms behind some magnetic relaxation behavior remain unclear even in common magnetic materials. For example, $\mathrm{CoFe}$ alloys were recently theoretically predicted [12] and experimentally demonstrated [13] to display ultralow damping which was previously believed to be unachievable in metallic ferromagnets. In this article, inspired by recent theoretical and experimental findings related to spin pumping [14-19], we chose to investigate bare permalloy $(\mathrm{NiFe})$ in an attempt to determine the incompletely understood origin of their nonmonotonous temperature dependence of ferromagnetic damping [20-25]. More specifically, typical $3 d$ transition metals $(\mathrm{Co}, \mathrm{Ni}, \mathrm{Fe})$ and associated alloys (including $\mathrm{NiFe}$ ) frequently show a minimum in the temperature dependence of their damping [26,27]. It is now accepted that a conductivitylike term related to intraband scattering dominates local intrinsic damping at low temperatures, whereas a resistivitylike term due to interband scattering takes over at higher temperatures [27]. Sometimes for $\mathrm{NiFe}$, a contrasting pronounced maximum was unexpectedly observed in the temperaturedependent damping [20]. This finding, and the reasons for it, remain controversial and are still being discussed. It has been suggested that the temperature-dependent reorientation of $\mathrm{NiFe}$ surface spins from in plane to out of plane could account for the maximum damping observed [22-24]. However, recent evidence indicates that spin reorientation may occur at a much lower temperature than the maximum damping [28]. An alternative mechanism was also proposed involving slow

\footnotetext{
*vincent.baltz@cea.fr
}

relaxation on paramagnetic impurities present in, or adjacent to, the oscillating ferromagnetic material $[20,21,29,30]$. In this process, the oscillations in the magnetization of the ferromagnet modulate how the energy splits between impurity levels. Subsequent relaxation of the impurities influences ferromagnetic damping. In fact, if not protected from oxidation due to exposure to air, a few monolayers of the NiFe layer will naturally oxidize to form a passivating oxide layer $(\mathrm{NiFeOx})$. This layer contains a complex mixture of $\mathrm{NiO}$ and $\mathrm{FeO}$ antiferromagnetic alloys with variable stoichiometry gradients [25]. In this context, the potential influence of relaxation of interface paramagnetic impurities in bilayers where a ferromagnet is exchange biased to an antiferromagnet was considered in several studies [31-34]. However, the results of these studies led to divergent mechanisms being presented to explain the temperature dependence of the relaxation rate for impurities [31-34]. Beyond paramagnetic impurities or exchange-bias interactions, the presence of NiFe antiferromagnetic surface oxides raises the question of how spin angular momentum is absorbed by the antiferromagnetic layer itself $[35,36]$. In this process, transfer/sink and propagation of spin angular momentum involves magnons from the oscillating ferromagnet feeding into the antiferromagnet, due to magnetic coupling [37,38]. The end result is an overall enhancement of the total damping of the ferromagnet $[35,36]$. In addition, near the phase transition for the magnetic order of the antiferromagnetic layer, i.e., around its Néel temperature, the magnetic fluctuations were shown to lead to a maximum spin-pumping efficiency [16-18]. The origin of this phenomenon was corroborated by calorimetry $[16,39]$ and neutron diffraction measurements [18].

In this work, we investigated whether enhanced spin angular momentum absorption at the magnetic phase transition of native interface- and surface-oxidized layers could be an alternative mechanism explaining the temperature-dependent ferromagnetic relaxation of bare $\mathrm{NiFe}$. We examined temperature-dependent ferromagnetic relaxation in $\mathrm{NiFe}$ thin films, and how it was affected by native oxidation of the $\mathrm{NiFe}$ layer and the number of native oxide layers surrounding the $\mathrm{NiFe}$ (two, one, or none). Spin-pumping experiments were performed at various temperatures on a first series of samples consisting of $\mathrm{Si} / \mathrm{SiO}_{2}(500) / \mathrm{NiFe}(8)$ (short name: $\mathrm{SiO}_{2} / \mathrm{NiFe}$ ), $\mathrm{Si} / \mathrm{SiO}_{2}(500) / \mathrm{NiFe}(8) / \mathrm{Cu}(3) / \mathrm{Al}(2)$ (short name: $\mathrm{SiO}_{2} / \mathrm{NiFe} / \mathrm{Cu} / \mathrm{Al}$ ), $\mathrm{Si}_{/} \mathrm{SiO}_{2}(500) / \mathrm{Cu}(6) / \mathrm{NiFe}(8)$ 
(a)

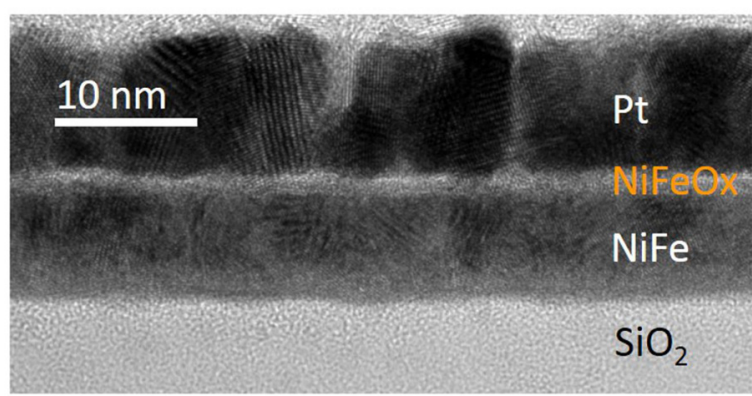

(b)

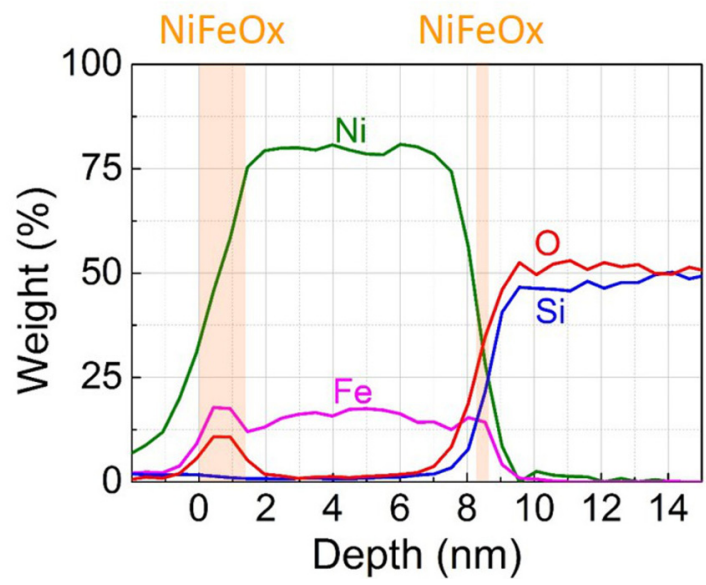

FIG. 1. (a) Transmission electron microscopy image (TEM) and (b) energy-dispersive x-ray spectroscopy (EDX) data for $\mathrm{SiO}_{2} / \mathrm{NiFe}$ sample. Samples were capped with $\mathrm{Pt}$ in preparation for the TEM experiment.

(short name: $\mathrm{SiO}_{2} / \mathrm{Cu} / \mathrm{NiFe}$ ), and $\mathrm{Si} / \mathrm{SiO}_{2}(500) / \mathrm{Cu}(6) / \mathrm{NiFe}(8)$ $/ \mathrm{Cu}(3) / \mathrm{Al}(2)$ (short name: $\mathrm{SiO}_{2} / \mathrm{Cu} / \mathrm{NiFe} / \mathrm{Cu} / \mathrm{Al}$ ) multilayers. All thicknesses are given in nanometers. Stacks were deposited on thermally oxidized silicon substrates $\left[\mathrm{Si} / \mathrm{SiO}_{2}(500)\right]$ at room temperature by dc-magnetron sputtering. The $\mathrm{NiFe}$ layer was deposited from a permalloy target $\left[\mathrm{Ni}_{81} \mathrm{Fe}_{19}\right.$ (at.\%)]. An $\mathrm{Al}(2)$ cap was added, forming a protective passivating AlOx film, to block oxidization by air in some samples. The other samples were exposed to air. Data recorded after a week and after a month overlapped, indicating that oxidation has reached a maximum after a week. Uncapped layers were therefore exposed to air for a minimum of one week before any measurements were performed. Transmission electron microscopy (TEM) analysis [Fig. 1(a)] was used to view oxidation of the $\mathrm{NiFe}$ layer in the $\mathrm{SiO}_{2} / \mathrm{NiFe}$ stack. After exposure to air, some sample pieces were capped with $\mathrm{Pt}$ in preparation for the TEM experiment. This cap protects the sample's surface from damage during the thinning and polishing steps required for TEM. It also enhances the TEM contrast around the sample surface. Results of the TEM investigations indicated a NiFe surface oxide $(\mathrm{NiFeOx})$ produced by $\mathrm{NiFe}$ oxidation in air. The thickness of the NiFeOx surface oxide, as determined from the TEM data was approximately $1.6 \pm 0.2 \mathrm{~nm}$. The margin of error corresponds to typical errors in thickness measurements performed at various locations in TEM images. The $\mathrm{NiFeOx}$ thickness value is in line with data from the literature, where passivating surface oxides were reported to measure nanometers thick [25]. Results from energy-dispersive x-ray spectroscopy (EDX) measurements [Fig. 1(b)] confirm the presence of a surface-oxidized layer and reveal the presence of another native oxidized layer at the interface between the $\mathrm{SiO}_{2}$ and NiFe layers. This lower oxide layer was not visible in the TEM image due to a lack of contrast with the $\mathrm{SiO}_{2}$ underlayer. Unlike the top oxide, this bottom oxide is not produced due to oxidation in air. Rather, it forms naturally at the $\mathrm{SiO}_{2} / \mathrm{NiFe}$ interface, likely activated by the $\mathrm{Ni}$ and $\mathrm{Fe}$ atoms when they interact with the $\mathrm{SiO}_{2}$ surface during sputter deposition. The presence and thickness (around $0.3 \pm 0.2 \mathrm{~nm}$ ) of this bottom oxide layer was determined from the horizontal shift in the oxygen and silicon traces in EDX data [visible in Fig. 1(b)]. Indeed, Fig. 1(b) represents atomic weight as a function of sample depth. Since the O signal rises before the Si signal, some oxygen atoms must have mixed with the $\mathrm{NiFe}$ layer. The shift between the $\mathrm{Si}$ and $\mathrm{O}$ traces along the sample depth corresponds to the thickness of this bottom NiFeOx layer. The margin of error corresponds to typical errors reported for EDX data due to measurements performed at different locations in the film. From the EDX data, we also calculated that in the $\mathrm{SiO}_{2} / \mathrm{NiFe}$ sample, the $\mathrm{Ni}$ and $\mathrm{Fe}$ atoms extend over a total thickness of around $8.1 \pm 0.2 \mathrm{~nm}$. Complementary EDX measurements performed on $\mathrm{SiO}_{2} / \mathrm{NiFe} / \mathrm{Cu} / \mathrm{Al}$ sample, where the NiFe layer was not air oxidized, indicated that the $\mathrm{Ni}$ and $\mathrm{Fe}$ atoms also extend over a total thickness of around $8 \pm 0.3 \mathrm{~nm}$. This observation suggests negligible expansion of the lattice parameter for the oxide layer in $\mathrm{SiO}_{2} / \mathrm{NiFe}$ samples. The margins of error reported here give an estimate of the typical error level between nominal and actual thickness. To sum up, from the TEM and EDX experiments we can conclude that the initial $\mathrm{SiO}_{2} / \mathrm{NiFe}, \mathrm{SiO}_{2} / \mathrm{NiFe} / \mathrm{Cu} / \mathrm{Al}$, $\mathrm{SiO}_{2} / \mathrm{Cu} / \mathrm{NiFe}$, and $\mathrm{SiO}_{2} / \mathrm{Cu} / \mathrm{NiFe} / \mathrm{Cu} / \mathrm{Al}$ multilayers are in fact the following stacks $\mathrm{SiO}_{2} / \mathrm{NiFeOx}(0.3) / \mathrm{NiFe} / \mathrm{NiFeOx}$ (1.6), $\quad \mathrm{SiO}_{2} / \mathrm{NiFeOx}(0.3) / \mathrm{NiFe} / \mathrm{Cu} / \mathrm{AlOx}, \quad \mathrm{SiO}_{2} / \mathrm{Cu} / \mathrm{NiFe} /$ $\mathrm{NiFeOx}(1.6)$, and $\mathrm{SiO}_{2} / \mathrm{Cu} / \mathrm{NiFe} / \mathrm{Cu} / \mathrm{AlOx}$, respectively.

We next investigated the magnetic nature of the surfaceoxidized layers by measuring hysteresis loops at various temperatures using a magnetometer [Fig. 2(a)]. These results show a loop shift $\left(\mathrm{H}_{\mathrm{E}}\right)$ along the axis of the magnetic field, demonstrating magnetic exchange-bias interactions [40,41] between the $\mathrm{NiFe}$ ferromagnetic layer and the $\mathrm{NiFeOx}$ surfaceoxidized layer. These data confirmed the antiferromagnetic nature of the top surface-oxidized layer. The data presented in Fig. 2(b) further indicated that $\mathrm{H}_{\mathrm{E}}$ decreases as the temperature rises. The ferromagnetic/antiferromagnetic blocking temperature $\left(T_{\mathrm{B}}\right)$ can be extracted from $\mathrm{H}_{\mathrm{E}}$ vs $T$ by determining the temperature at which $\mathrm{H}_{\mathrm{E}}$ vanishes $[40,41] . T_{B}$ is expected to be much smaller than the critical temperature $\left(T_{\text {crit }}\right)$ for the antiferromagnetic-to-paramagnetic transition [40,41], and for exchange-bias interactions with the top $\mathrm{NiFeOx}(1.6)$ layer, it was found to be about $15 \mathrm{~K}$ (see data for the $\mathrm{SiO}_{2} / \mathrm{Cu} / \mathrm{NiFe}$ and $\mathrm{SiO}_{2} / \mathrm{NiFe}$ samples). This relationship can be explained as $T_{B}$ is linked to the interfacial exchange interactions between the ferromagnet and the antiferromagnet, whereas $T_{\text {crit }}$ relates to the exchange stiffness between all antiferromagnetic moments. For exchange-bias interactions with the lower $\mathrm{NiFeOx}(0.3)$ layer (see data for the $\mathrm{SiO}_{2} / \mathrm{NiFe} / \mathrm{Cu}$ sample), $T_{B}$ was determined to be sub-K. Its value could not be measured based on the data shown in Fig. 2(b) due to the fact that 


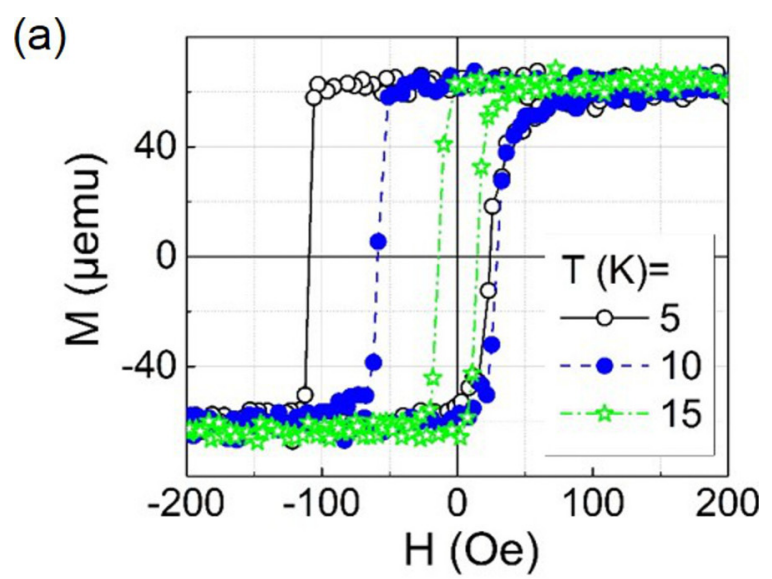

(b)

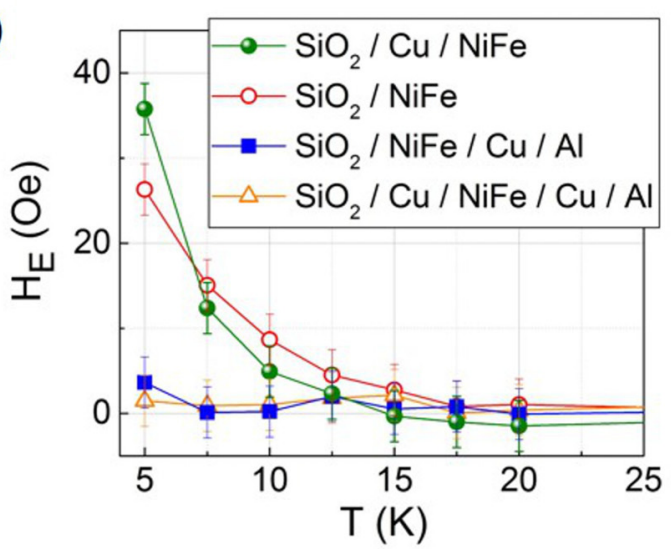

FIG. 2. (a) Representative magnetization (M) vs field (H) hysteresis loops at different temperatures for a $\mathrm{SiO}_{2} / \mathrm{Cu} / \mathrm{NiFe}$ sample. (b) Temperature $(T)$ dependence of the hysteresis loop shift $\left(\mathrm{H}_{\mathrm{E}}\right)$.

the lower $\mathrm{NiFeOx}(0.3)$ oxide layer is very thin and displays a reduced $T_{\text {crit }}$. Note that for the ultrathin $\mathrm{NiFeOx}(0.3)$ layer, $T_{\text {crit }}$ probably describes a frozen-to-liquid spin transition. Results confirming the reduced value of $T_{\text {crit }}$ will be discussed below.

Spin-pumping experiments [Fig. 3(a)] and related series of ferromagnetic resonance spectra were recorded for temperatures $(T)$ ranging between 20 and $300 \mathrm{~K}$, using a continuouswave electron paramagnetic resonance spectrometer operating at $9.6 \mathrm{GHz}$ fitted with a cavity. For each temperature the Gilbert damping $(\alpha)$ was determined by fitting the $\mathrm{NiFe}$ resonance spectrum to a Lorenzian. The value of $\alpha$ was extracted from $\alpha(T)=\left[\Delta H_{p p}(T)-\Delta H_{0}(300 \mathrm{~K})\right] \sqrt{3}|\gamma| /(2 \omega)$, where $\Delta H_{p p}$ is the peak-to-peak linewidth for the spectrum, $\gamma$ is the gyromagnetic ratio, and $\omega$ is the angular frequency [42]. $\Delta H_{0}$ relates to spatial variations in the magnetic properties. This parameter was determined from standard $\Delta H_{p p}$ vs $\omega / 2 \pi$ plots using a separate, broadband coplanar waveguide operating at room temperature for frequencies ranging between 2 and 24 $\mathrm{GHz}$ [42]. For bare NiFe films similar to those described above, $\Delta H_{0}$ was previously found to be a temperatureinvariant parameter [43]. Figure 3(b) shows $\alpha$ plotted against temperature. The pronounced maximum at $T=70 \mathrm{~K}$ corresponds to the top $\mathrm{NiFeOx}(1.6)$ layer resulting from natural oxidation in air (see data for the $\mathrm{SiO}_{2} / \mathrm{NiFe}$ and $\mathrm{SiO}_{2} / \mathrm{Cu} / \mathrm{NiFe}$ samples). The amplitude of this maximum was threefold the amplitude measured at $300 \mathrm{~K}$. A less pronounced contribution (a)
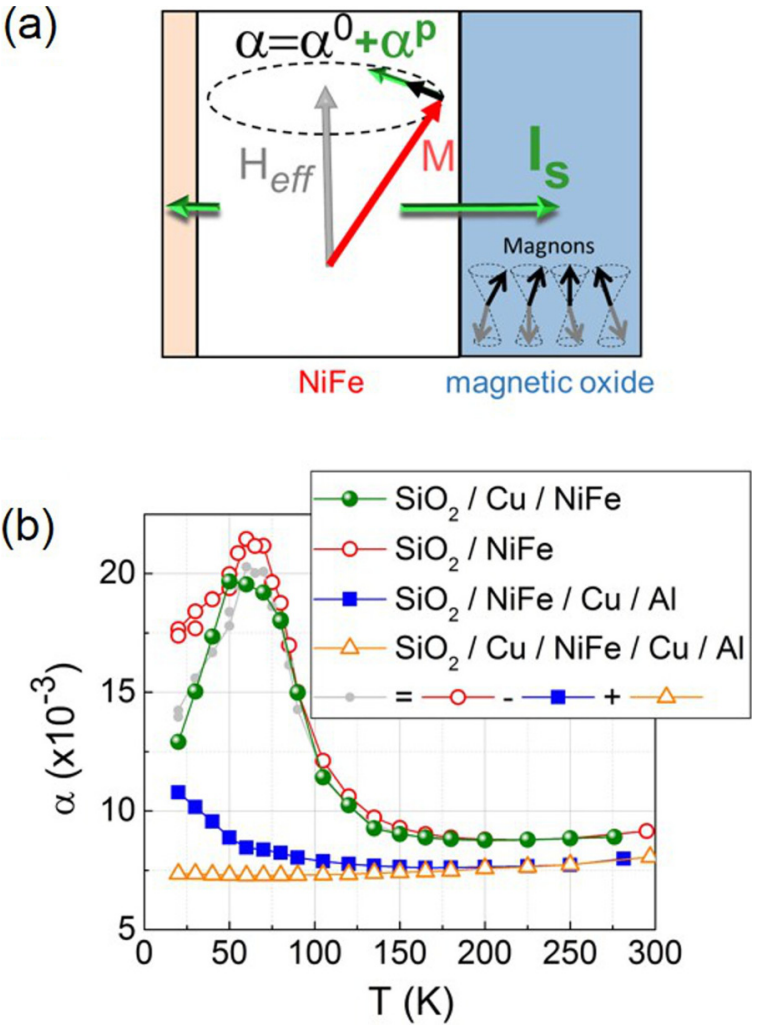

FIG. 3. (a) Diagrammatic representation of the spin-pumping experiment. (b) Temperature $(T)$ dependence of the NiFe layer Gilbert damping $(\alpha)$. The NiFe layer is surrounded by two, one, or no native oxide layers. When the $\mathrm{NiFe}$ is deposited directly on $\mathrm{SiO}_{2}$ a 0.3 -nm-thick $\mathrm{NiFeOx}$ naturally forms at the $\mathrm{SiO}_{2} / \mathrm{NiFe}$ interface, activated by the $\mathrm{Ni}$ and $\mathrm{Fe}$ atoms when interacting with the $\mathrm{SiO}_{2}$ surface during sputter deposition. When the NiFe layer is left uncapped it naturally undergoes oxidation due to contact with air, resulting in a 1.6-nm-thick $\mathrm{NiFeOx}$ surface layer.

is visible at lower temperatures in samples containing the bottom $\mathrm{NiFeOx}(0.3)$ layer, where the $\mathrm{NiFe}$ become naturally oxidized due to contact with the $\mathrm{SiO}_{2}$ layer (see data for the $\mathrm{SiO}_{2} / \mathrm{NiFe} / \mathrm{Cu} / \mathrm{Al}$ sample). When the $\mathrm{NiFe}$ layer was isolated from oxygen atoms on both sides (in the $\mathrm{SiO}_{2} / \mathrm{Cu} / \mathrm{NiFe} / \mathrm{Cu} / \mathrm{Al}$ sample) no such maxima were observed.

Since the oxidized layers are magnetic, the NiFe damping is the sum of local intrinsic damping $\left(\alpha^{0}\right)$ that can be described by intraband and interband scattering [27] and additional nonlocal damping $\left(\alpha^{\mathrm{p}, \mathrm{i}}\right)$ associated with the surface/interface $\mathrm{NiFeOx}$ oxide(s) acting as a spin absorber for angular momentum. The temperature dependence of $\alpha$ can thus be expressed as $\alpha(T)=\alpha^{0}(T)+\sum_{i} \alpha^{p, i}(T)[14,35,36]$, where $i$ accounts for the uppermost and/or lowermost NiFeOx spin absorber. Data obtained with the $\mathrm{SiO}_{2} / \mathrm{Cu} / \mathrm{NiFe} / \mathrm{Cu} / \mathrm{Al}$ sample (no spin absorber) give the temperature dependence of the local intrinsic NiFe Gilbert damping $\left[\alpha_{\mathrm{SiO}_{2} / \mathrm{Cu} / \mathrm{NiFe} / \mathrm{Cu} / \mathrm{Al}}(T)=\alpha^{0}(T)\right]$ with a detectable conductivitylike to resistivitylike progression [26,27]. From Fig. 3(b), we can thus conclude that the temperature dependence of $\alpha^{0}$ can be neglected, but that $\alpha^{\mathrm{p}, \mathrm{i}}$ is highly temperature dependent. The nonlocal damping is related to the spin mixing conductance across the $\mathrm{NiFe} / \mathrm{NiFeOx}(1.6)$ and/or $\mathrm{NiFeOx}(0.3) / \mathrm{NiFe}$ interfaces 
$\left(g_{s}^{i}\right)$ as presented in [14] by $\alpha^{p, i}(T)=\frac{1}{S_{0} N_{\mathrm{SI}}} g_{s}^{i}(T)$, where $S_{0}$ is the norm of the spin operator, and $N_{\mathrm{SI}}$ is the number of lattice sites in the NiFe spin injector (SI). The spin mixing conductance across the interface is linked to the magnetic susceptibility of the spin absorber [14] via $g_{s}^{i}(T)=\frac{2 J_{S d}^{2} S_{0}^{2} N_{\mathrm{int}}}{\hbar^{2} N_{\mathrm{SA}}^{i}} \sum_{k} \frac{1}{\Omega_{r f}} \operatorname{Im} \chi_{k}^{R, i}\left(\Omega_{r f}, T\right)$, where $N_{\mathrm{int}}$ is the number of spins localized at the interface, $N_{\mathrm{SA}}^{i}$ is the number of lattice sites in the spin absorber (SA) $i, J_{s d}$ is the $s-d$ exchange interaction at the SI/SA interface, $k$ is the wave vector, and $\Omega_{r f}$ is the angular frequency of NiFe at resonance. The temperature-dependent dynamic spin susceptibility of the spin absorber can be represented by $\chi_{k}^{R, i}\left(\Omega_{r f}, T\right)$. As a result, the nonlocal damping is given by $\alpha^{p, i}(T)=$ $\frac{2 J_{S d}^{2} S_{0} N_{\text {int }}}{\hbar^{2} N_{\mathrm{SA}}^{i} N_{\mathrm{SI}}} \sum_{k} \frac{1}{\Omega_{r f}} \operatorname{Im} \chi_{k}^{R, i}\left(\Omega_{r f}, T\right)$. The spin susceptibility of antiferromagnetic materials is known to display a maximum around the critical temperature for the magnetic phase transition due to magnetic fluctuations. This transition results in enhanced spin mixing conductance across the interface $\left(g_{s}^{i}\right)$. In other words, magnetic fluctuations in the spin absorber open more conduction channels across the interface, which translates into enhanced spin angular momentum absorption $\left(\alpha^{\mathrm{p}, \mathrm{i}}\right)$ and therefore into maximal $\mathrm{NiFe}$ total damping $(\alpha)$, as observed in Fig. 3(b). From data for $\mathrm{SiO}_{2} / \mathrm{Cu} / \mathrm{NiFe}$, where $\alpha_{\mathrm{SiO}_{2} / \mathrm{Cu} / \mathrm{NiFe}}(T)=\alpha^{0}(T)+\alpha^{p, \mathrm{NiFeOx}(1.6)}(T)$, we deduced the Néel temperature for the magnetic phase transition of the top 1.6-nm-thick $\mathrm{NiFeOx}$ oxide, at approximately $70 \mathrm{~K}$. From the $\mathrm{SiO}_{2} / \mathrm{NiFe} / \mathrm{Cu} / \mathrm{Al}$ in Fig. 3(b), where $\alpha_{\mathrm{SiO}_{2} / \mathrm{NiFe} / \mathrm{Cu} / \mathrm{Al}}(T)=\alpha^{0}(T)+\alpha^{p, \mathrm{NiFeOx}(0.3)}(T)$, we concluded that the critical temperature for the phase transition of the lowermost 0.3 -nm-thick $\mathrm{NiFeOx}$ oxide, which formed naturally at the interface between the $\mathrm{NiFe}$ and $\mathrm{SiO}_{2}$ layers, is less than $20 \mathrm{~K}$. We infer that this temperature is actually well below $20 \mathrm{~K}$, and probably sub-K since the amplitude of the damping peak for the 0.3 -nm-thick $\mathrm{NiFeOx}$ oxide is expected to be fivefold $(1.6 / 0.3)$ that of the 1.6-nm-thick oxide. This difference can be explained by the fact that $\alpha^{\mathrm{p}}$ is inversely proportional to the number of lattice sites in the spin absorber $\left(N_{\mathrm{SA}}\right)$. Finally, data for the $\mathrm{SiO}_{2} / \mathrm{NiFe}$ sample relate to $\alpha_{\mathrm{SiO}_{2} / \mathrm{NiFe}}(T)=\alpha^{0}(T)+\alpha^{p, \mathrm{NiFeOx}(0.3)}(T)+\alpha^{p, \mathrm{NiFeOx}(1.6)}(T)$. From the four relations above between $\alpha_{\mathrm{SiO}_{2} / \mathrm{NiFe}}$, $\alpha_{\mathrm{SiO}_{2} / \mathrm{NiFe} / \mathrm{Cu} / \mathrm{Al} l}, \alpha_{\mathrm{SiO}_{2} / \mathrm{Cu} / \mathrm{NiF} / \mathrm{Cu} / \mathrm{Al}}$, and $\alpha_{\mathrm{SiO}_{2} / \mathrm{Cu} / \mathrm{NiFe}} ;$ and $\alpha^{0}$, $\alpha^{p, \mathrm{NiFeOx}(0.3)}$ and $\alpha^{p, \mathrm{NiFeOx}(1.6)}$ our experimental data should confirm the following equation: $\alpha_{\mathrm{SiO}_{2} / \mathrm{NiFe}}-\alpha_{\mathrm{SiO}_{2} / \mathrm{NiFe} / \mathrm{Cu} / \mathrm{Al}}+$ $\alpha_{\mathrm{SiO}_{2} / \mathrm{Cu} / \mathrm{NiFe} / \mathrm{Cu} / \mathrm{Al}}=\alpha_{\mathrm{SiO}_{2} / \mathrm{Cu} / \mathrm{NiFe}}$. The data shown in Fig. 3(b) clearly confirm the equation, where the small gray filled circles $\left(\alpha_{\mathrm{SiO}_{2} / \mathrm{NiFe}}-\alpha_{\mathrm{SiO}_{2} / \mathrm{NiFe} / \mathrm{Cu} / \mathrm{Al}}+\alpha_{\mathrm{SiO}_{2} / \mathrm{Cu} / \mathrm{NiFe} / \mathrm{Cu} / \mathrm{Al}}\right)$ satisfactorily overlap the green circles $\left(\alpha_{\mathrm{SiO}_{2} / \mathrm{Cu} / \mathrm{NiFe}}\right)$. This verification further supports absorption of spin angular momentum at the magnetic phase transition of the native surface-oxidized layers as an alternative mechanism explaining the temperature-dependent ferromagnetic relaxation of bare $\mathrm{NiFe}$, while also indicating satisfactory reproducibility of data and native oxidation from sample to sample.

We note here that it is essential that transfer and propagation of spin angular momentum directly involves magnonic transport. In other words, the $\mathrm{NiFeOx}$ must be fed by spin waves through direct magnetic coupling with NiFe. The difference in spin-pumping efficiency by a

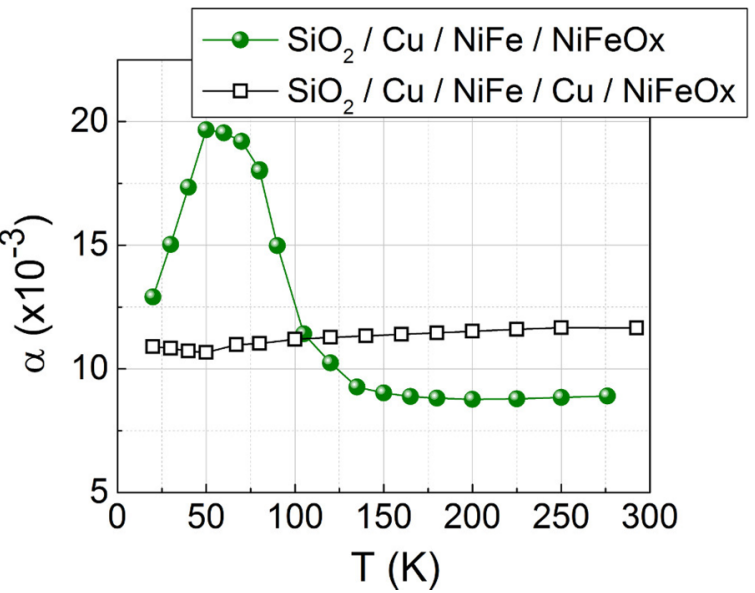

FIG. 4. Temperature $(T)$ dependence of damping $(\alpha)$ of a $\mathrm{NiFe}$ ferromagnet directly coupled to a 1.6-nm-thick antiferromagnetic $\mathrm{NiFeOx}$ or separated by a 3 -nm-thick $\mathrm{Cu}$ layer. In the $\mathrm{SiO}_{2} / \mathrm{Cu} / \mathrm{NiFe} / \mathrm{NiFeOx}$ sample, $\mathrm{NiFeOx}$ results from the native oxidation of $\mathrm{NiFe}(8 \mathrm{~nm})$, creating a passivating 1.6-nm-thick layer. In the $\mathrm{SiO}_{2} / \mathrm{Cu} / \mathrm{NiFe} / \mathrm{Cu} / \mathrm{NiFeOx}$ sample, $\mathrm{NiFeOx}$ results from complete native oxidation of a $\mathrm{NiFe}(1.6 \mathrm{~nm})$ layer.

$\sim 1.6$-nm-thick antiferromagnetic $\mathrm{NiFeOx}$ directly coupled to $\mathrm{NiFe}$, or separated from the ferromagnetic $\mathrm{NiFe}$ layer by a 3-nm-thick $\mathrm{Cu}$ layer is shown in Fig. 4. The enhanced damping at $70 \mathrm{~K}$ due to the magnetic phase transition of the $\mathrm{NiFeOx}$ is clearly observed in the case of direct magnonic transport but is suppressed when the $\mathrm{Cu}$ breaks the direct magnetic interaction between $\mathrm{NiFe}$ and $\mathrm{NiFeOx}$. In the latter case, spin transport is mediated by a purely electronic transport regime through $\mathrm{Cu}$. As the spin diffusion length for $\mathrm{Cu}$ is much longer than $3 \mathrm{~nm}$, spin propagation will not be altered by passing through this layer. However, from the data shown in Fig. 4 virtually no spin angular momentum is transmitted to the $\mathrm{NiFeOx}$. The presence of intermediate copper oxide at the $\mathrm{Cu} / \mathrm{NiFeOx}$ interface cannot be excluded and may also account for the overall increase of $\alpha$.

We further investigated how $T_{\text {crit }}$ is influenced by the thickness of the native oxide. Spin-pumping experiments were performed at various temperatures on a second series of samples, where $\mathrm{NiFe}\left(t_{\mathrm{NiFe}}\right)$ layers were grown on $\mathrm{Si} / \mathrm{SiO}_{2}$, naturally oxidized by air for a week before adding another $\mathrm{NiFe}(8)$ layer, which was left uncapped. $t_{\mathrm{NiFe}}$ is the thicknesses of the bottom NiFe layer $(0.5,1$, or $1.5 \mathrm{~nm})$. Based on the results presented above, the lowermost $\mathrm{NiFe}$ layer is expected to be fully oxidized in air. The samples therefore consisted of a $\mathrm{Si} / \mathrm{SiO}_{2} / \mathrm{NiFeOx}\left(t_{\mathrm{NiFeO}}\right) / \mathrm{NiFe} / \mathrm{NiFeOx}(1.6)$ with nominal $t_{\mathrm{NiFeOx}}=0.5,1$, or $1.5 \mathrm{~nm}$. The NiFe layer is therefore influenced by two spin angular momentum absorbers, and its damping will correspond to the sum of local intrinsic damping, nonlocal extrinsic damping due to spin absorption by the lower $\mathrm{NiFeOx}\left(t_{\mathrm{NiFeOx}}\right)$, and nonlocal extrinsic damping due to spin absorption by the upper $\mathrm{NiFeOx}(1.6)$ layer: $\alpha(T)=$ $\alpha^{0}(T)+\alpha^{p, \text { NiFeOx }\left(t_{\mathrm{NiFeO}}\right)}(T)+\alpha^{p, \mathrm{NiFeOx}(1.6)}(T)$. Due to fluctuations of the antiferromagnetic order, $\alpha^{p, \operatorname{NiFeOx}\left(t_{\mathrm{NiFeO}}\right)}(T)$ and $\alpha^{p, \mathrm{NiFeOx}(1.6)}(T)$ are expected to show a maximum at the magnetic phase transition for the $\mathrm{NiFeOx}\left(t_{\mathrm{NiFeOx}}\right)$ and $\mathrm{NiFeOx(1.6)} \mathrm{layer,} \mathrm{respectively.} \mathrm{Figure} \mathrm{5(a)} \mathrm{shows} \alpha$ plotted 

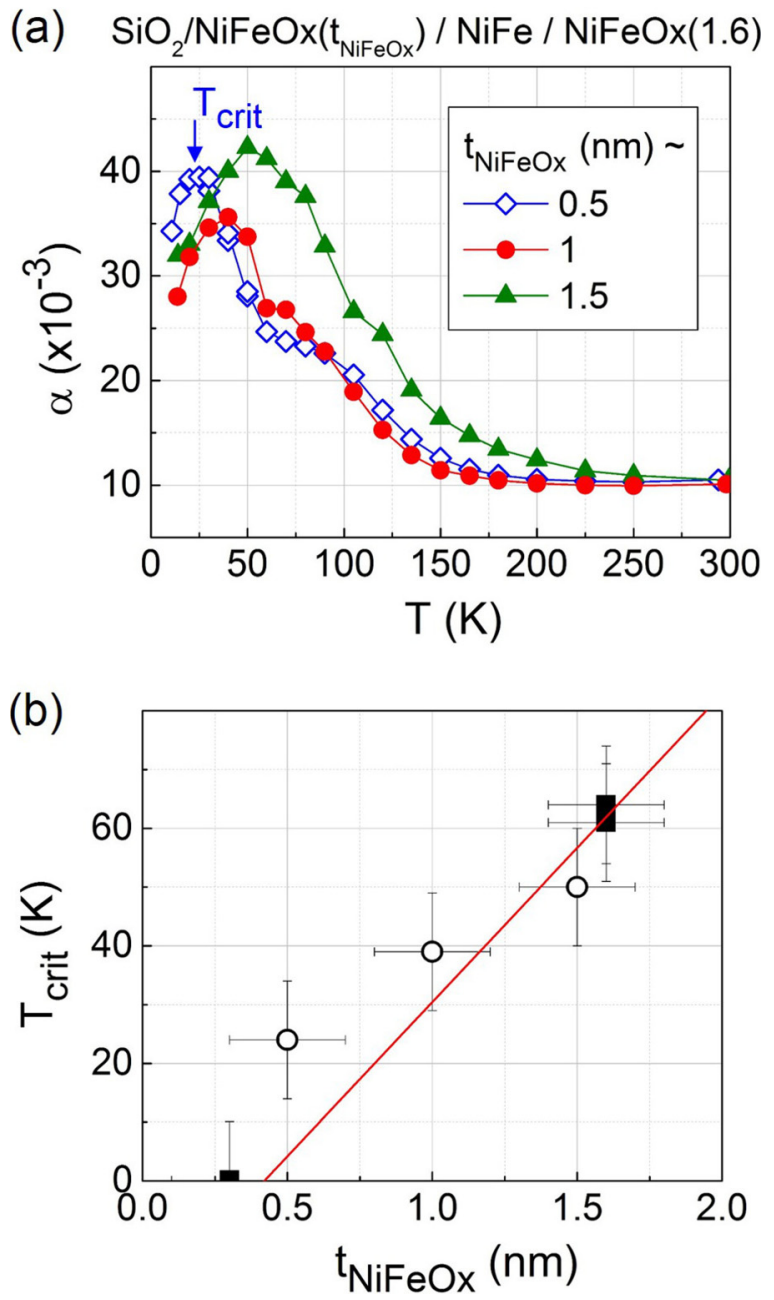

FIG. 5. (a) Temperature ( $T$ ) dependence of the Gilbert damping $(\alpha)$ of the $\mathrm{NiFe}$ layer on temperature $(T)$ in $\mathrm{SiO}_{2} / \mathrm{NiFeOx}\left(t_{\mathrm{NiFeOx}}\right) / \mathrm{NiFe} / \mathrm{NiFeOx}(1.6) \quad(\mathrm{nm})$ multilayers. (b) Thickness dependence of the critical temperature $\left(T_{\text {crit }}\right)$ for the magnetic phase transition of the oxidized NiFe layer. Open circles represent data deduced from (a). Full squares represent data deduced from Fig. 3(b). Line fitting was based on the equation presented by Zhang et al. [44] in the thin-layer regime for a $(\mathrm{NiO})_{81}(\mathrm{FeO})_{19}$ alloy.

against temperature for these multilayers. The data indicate two contributions to $\alpha$ for samples containing the 0.5and 1-nm-thick lowermost $\mathrm{NiFeOx}$ layers. In line with the results presented above, the contribution (shoulder) at around $70 \mathrm{~K}$ corresponds to the magnetic phase transition of the uppermost $\mathrm{NiFeOx}(1.6)$ layer. The expected maximum in damping actually overlaps with the tail of the peak that can be observed at lower temperatures (at around 25 and $40 \mathrm{~K}$ for $t_{\mathrm{NiFeOx}}=0.5$ and $1 \mathrm{~nm}$, respectively). The peak at the lower temperature corresponds to the magnetic phase transition of the lowermost $\mathrm{NiFeOx}(0.5$ or 1) layer. From Fig. 5(a), we observe that the contribution of the phase transition of the lower layer shifts towards higher temperatures as its thickness increases. With the $\mathrm{SiO}_{2} / \mathrm{NiFeOx}(1.5) / \mathrm{NiFe} / \mathrm{NiFeOx}(1.6)$ sample, the NiFe is sandwiched between two similar
$\mathrm{NiFeOx}$ layers. The top and bottom $\mathrm{NiFeOx}$ layers in the $\mathrm{Si} / \mathrm{SiO}_{2} / \mathrm{NiFeOx}(1.5) / \mathrm{NiFe} / \mathrm{NiFeOx}(1.6)$ sample therefore absorb similar amounts of spin current on both sides $\left[\alpha^{p, \mathrm{NiFeOx}(1.5)}(T) \sim \alpha^{p, \mathrm{NiFeOx}(1.6)}(T)\right]$ and share a similar $T_{\text {crit }}$, around $70 \mathrm{~K}$, as previously deduced for the $\mathrm{NiFeOx}(1.6)$ layer [Fig. 3(b)]. As a result, the peaks corresponding to the magnetic phase transitions for the top $\mathrm{NiFeOx}(1.5)$ and bottom $\mathrm{NiFeOx}(1.6)$ layers overlapped. The peak's amplitude for the $\mathrm{Si} / \mathrm{SiO}_{2} / \mathrm{NiFeOx}(1.5) / \mathrm{NiFe} / \mathrm{NiFeOx}(1.6)$ sample with two similar spin absorbers [Fig. 5(a)] was effectively close to twice the amplitude of the peak for the $\mathrm{SiO}_{2} / \mathrm{Cu} / \mathrm{NiFe} / \mathrm{NiFeOx}(1.6)$ sample [nominal $\mathrm{SiO}_{2} / \mathrm{Cu} / \mathrm{NiFe}$ in Fig. 3(b)]. Satisfactory reproducibility of data and native oxidation can also be concluded from these data. Figure 5(b) illustrates how the critical temperature for the $\mathrm{NiFeOx}$ layer, regardless of its actual nature, is directly proportional to its thickness. This linear relationship is in line with theories on finite size scaling of magnetic phase transitions $[44,45]$ whereby $T_{\text {crit }}\left(t_{\mathrm{NiFeOx}}\right)=$ $T_{\mathrm{N}}($ bulk $)\left(t_{\mathrm{NiFeOx}}-d\right) /\left(2 n_{0}\right)$, with $T_{\mathrm{N}}$ (bulk) as the bulk Néel temperature of the $\mathrm{NiFeOx}$ layer, $t_{\mathrm{NiFeOx}}$ as its thickness, $d$ as its lattice parameter, and $n_{0}$ as its phenomenological interspin correlation length. This corroboration of theory supports the fact that what we actually measured is the result of a magnetic phase transition of the $\mathrm{NiFeOx}$ layer, which was formed by natural oxidation of NiFe. Unfortunately, our data cannot be readily fitted to the model because the actual nature of the $\mathrm{NiFeOx}$ layer is complex, as it is composed of a mixture of different phases including $\mathrm{NiO}$ and $\mathrm{FeO}$ alloys, and variations in thickness due to the oxidation rate [25], nor can cluster formations be excluded. The red line in Fig. 4(b) represents a fit for the $\mathrm{Ni}_{81} \mathrm{Fe}_{19} \mathrm{Ox}$ layer determined by considering it as a $(\mathrm{NiO})_{81}(\mathrm{FeO})_{19}$ alloy (approximately proportional to the initial Ni-to-Fe atomic ratio). We used $T_{\mathrm{N}}($ bulk $)=0.81 T_{\mathrm{N}, \mathrm{NiO}}($ bulk $)+0.19 T_{\mathrm{N}, \mathrm{FeO}}$ (bulk) for fitting, with $T_{\mathrm{N}, \mathrm{NiO}}($ bulk $)=520 \mathrm{~K}, T_{\mathrm{N}, \mathrm{FeO}}($ bulk) $=200 \mathrm{~K}[40], d=$ $0.81 d_{\mathrm{NiO}}+0.19 d_{\mathrm{FeO}}, d_{\mathrm{NiO}}=0.417 \mathrm{~nm}$, and $d_{\mathrm{FeO}}=0.433 \mathrm{~nm}$. This fit is shown to give the reader an idea of what such a simple and straightforward assumption would give. The fit agreed with our data to a satisfactory extent, and returned $n_{0}=4.4 \mathrm{~nm}$ (approximately ten monolayers), which is typical for ordered magnetic films [25].

In conclusion, the main contribution of this paper is the experimental evidence it presents supporting an alternative mechanism explaining the incompletely understood physical origin of the temperature-dependent ferromagnetic relaxation of bare permalloy. Our results demonstrated that the peak in temperature dependence of permalloy damping can be understood in terms of enhanced absorption of spin angular momentum at the antiferromagnetic-to-paramagnetic phase transition of its naturally oxidized surface layers. These findings open perspectives for further investigations, since a multitude of magnetic materials naturally form antiferromagnetic spin absorbers upon oxidation in air or when alloys form with elements contained in neighboring layers.

We acknowledge financial support from the French National Agency for Research (ANR JCJC ASTRONICS, Grant No. ANR-15-CE24-0015-01). We also thank M. GallagherGambarelli for critical reading of the manuscript. 
[1] L. Landau and E. Lifshitz, Phys. Z. Sowjetunion 8, 101 (1935).

[2] T. L. Gilbert, IEEE Trans. Magn. 40, 3443 (2004).

[3] I. Žutić, J. Fabian, and S. Das Sarma, Rev. Mod. Phys. 76, 323 (2004).

[4] T. Jungwirth, X. Marti, P. Wadley, and J. Wunderlich, Nat. Nanotechnol. 11, 231 (2016).

[5] V. Baltz, A. Manchon, M. Tsoi, T. Moriyama, T. Ono, and Y. Tserkovnyak, arXiv: 1606.04284.

[6] M. Farle, Rep. Prog. Phys. 61, 755 (1999).

[7] H. V. Gomonay and V. M. Loktev, Low Temp. Phys. 40, 22 (2014).

[8] A. Kirilyuk, A. V. Kimel, and T. Rasing, Rev. Mod. Phys. 82, 2731 (2010).

[9] D. C. Ralph and M. D. Stiles, J. Magn. Magn. Mater. 320, 1190 (2008).

[10] Spin Current, edited by S. Maekawa, S. O. Valenzuela, E. Saitoh, and T. Kimura, Series on Semiconductor Science and Technology (Oxford University Press, Oxford, 2012).

[11] B. Heinrich, Ultrathin Magnetic Structures III (Springer, New York, 2005).

[12] S. Mankovsky, D. Ködderitzsch, G. Woltersdorf, and H. Ebert, Phys. Rev. B 87, 014430 (2013).

[13] M. A. W. Schoen, D. Thonig, M. L. Schneider, T. J. Silva, H. T. Nembach, O. Eriksson, O. Karis, and J. M. Shaw, Nat. Phys. 12, 839 (2016).

[14] Y. Ohnuma, H. Adachi, E. Saitoh, and S. Maekawa, Phys. Rev. B 89, 174417 (2014).

[15] R. Khymyn, I. Lisenkov, V. S. Tiberkevich, A. N. Slavin, and B. A. Ivanov, Phys. Rev. B 93, 224421 (2016).

[16] L. Frangou, S. Oyarzun, S. Auffret, L. Vila, S. Gambarelli, and V. Baltz, Phys. Rev. Lett. 116, 077203 (2016).

[17] W. Lin, K. Chen, S. Zhang, and C. L. Chien, Phys. Rev. Lett. 116, 186601 (2016).

[18] Z. Qiu, J. Li, D. Hou, E. Arenholz, A. T. N'Diaye, A. Tan, K.-I. Uchida, K. Sato, Y. Tserkovnyak, Z. Q. Qiu, and E. Saitoh, Nat. Commun. 7, 12670 (2016).

[19] S. M. Rezende, R. L. Rodríguez-Suárez, and A. Azevedo, Phys. Rev. B 93, 054412 (2016).

[20] C. E. Patton and C. H. Wilts, J. Appl. Phys. 38, 3537 (1967).

[21] V. L. Safonov and H. N. Bertram, J. Appl. Phys. 94, 529 (2003).

[22] M. Diáz De Sihues, P. J. Silva, and J. R. Fermin, Phys. B 354, 361 (2004)

[23] J. F. Sierra, V. V. Pryadun, F. G. Aliev, S. E. Russek, M. GarcíaHernández, E. Snoeck, and V. V. Metlushko, Appl. Phys. Lett. 93, 172510 (2008).
[24] J. F. Sierra, V. V. Pryadun, S. E. Russek, M. García-Hernández, F. Mompean, R. Rozada, O. Chubykalo-Fesenko, E. Snoeck, G. X. Miao, J. S. Moodera, and F. G. Aliev, J. Nanosci. Nanotechnol. 11, 7653 (2011).

[25] R. A. Pollak and C. H. Bajorek, J. Appl. Phys. 46, 1382 (1975).

[26] S. M. Bhagat and P. Lubitz, Phys. Rev. B 10, 179 (1974).

[27] K. Gilmore, Y. U. Idzerda, and M. D. Stiles, Phys. Rev. Lett. 99, 027204 (2007).

[28] Y. Zhao, Q. Song, S.-H. Yang, T. Su, W. Yuan, S. S. P. Parkin, J. Shi, and W. Han, Sci. Rep. 6, 22890 (2016).

[29] V. L. Safonov and H. N. Bertram, Phys. Rev. B 61, R14893(R) (2000).

[30] A. M. Clogston, Bell Syst. Tech. J. 34, 739 (1955).

[31] P. Lubitz, J. J. Krebs, M. M. Miller, and S. Cheng, J. Appl. Phys. 83, 6819 (1998).

[32] P. Lubitz, M. Rubinstein, J. J. Krebs, and S. F. Cheng, J. Appl. Phys. 89, 6901 (2001).

[33] R. D. McMichael, C. G. Lee, M. D. Stiles, F. G. Serpa, P. J. Chen, and W. F. Egelhoff, J. Appl. Phys. 87, 6406 (2000).

[34] J. Dubowik, F. Stobiecki, I. Gościańska, Y. P. Lee, A. Paetzold, and K. Röll, Eur. Phys. J. B 45, 283 (2005).

[35] Y. Tserkovnyak, A. Brataas, and G. E. W. Bauer, Phys. Rev. Lett. 88, 117601 (2002).

[36] Y. Tserkovnyak, A. Brataas, G. E. W. Bauer, and B. I. Halperin, Rev. Mod. Phys. 77, 1375 (2005).

[37] C. Hahn, G. De Loubens, V. V. Naletov, J. Ben Youssef, O. Klein, and M. Viret, Europhys. Lett. 108, 57005 (2014).

[38] H. Wang, C. Du, P. C. Hammel, and F. Yang, Phys. Rev. Lett. 113, 097202 (2014).

[39] D. Petti, E. Albisetti, H. Reichlová, J. Gazquez, M. Varela, M. Molina-Ruiz, A. F. Lopeandía, K. Olejník, V. Novák, I. Fina, B. Dkhil, J. Hayakawa, X. Marti, J. Wunderlich, T. Jungwirth, and R. Bertacco, Appl. Phys. Lett. 102, 192404 (2013).

[40] J. Nogués and I. K. Schuller, J. Magn. Magn. Mater. 192, 203 (1999).

[41] A. E. Berkowitz and K. Takano, J. Magn. Magn. Mater. 200, 552 (1999)

[42] A. Ghosh, S. Auffret, U. Ebels, and W. E. Bailey, Phys. Rev. Lett. 109, 127202 (2012).

[43] Private communication. Data obtained by C. Cheng and W. E. Bailey of Columbia University, New York, for similar samples using a closed-cycle cryostat from 30 to $300 \mathrm{~K}$, fitted with broadband FMR (2-18 GHz).

[44] R. Zhang and R. F. Willis, Phys. Rev. Lett. 86, 2665 (2001).

[45] X. Y. Lang, W. T. Zheng, and Q. Jiang, Phys. Rev. B 73, 224444 (2006). 